\title{
Do Otimismo Explicativo ao Disposicional: a Perspectiva da Psicologia Positiva
}

\author{
Micheline Roat Bastianello - Universidade Federal do Rio Grande do Sul, Porto Alegre, Brasil \\ Claudio Simon Hutz - Universidade Federal do Rio Grande do Sul, Porto Alegre, Brasil
}

\begin{abstract}
Resumo
O otimismo, a partir da sabedoria popular, passando pelas discussões filosóficas até os estudos empíricos em Psicologia está fortemente associado a uma visão positiva da vida, especialmente diante de adversidades. O presente trabalho visa apresentar duas abordagens teóricas e de investigação empírica sobre o otimismo no campo da Psicologia Positiva. A perspectiva de Seligman define o otimismo como aprendido, relacionado aos estilos explicativos, enquanto Scheier e Carver compreendem o otimismo a partir de uma dimensão disposicional, baseado em expectativas generalizadas. No presente estudo, salientam-se as diferentes posições teóricas e metodológicas, os estudos realizados em diferentes culturas e com variáveis, como autoestima e personalidade.

Palavras-chave: Otimismo, Psicologia Positiva, personalidade, autoestima.
\end{abstract}

From Explanatory to Disposicional Optimism: a Perspective from Positive Psychology

\begin{abstract}
Optimism from the view of folk's wisdom, through the philosophical discussions to empirical studies in Psychology is strongly associated with a positive outlook on life, especially in light of disruptive events. This paper presents two theoretical approaches and empirical research on optimism in the field of Positive Psychology. The prospect of Martin Seligman defines optimism as learned, related to explanatory style, while Michael Scheier and Charles Carver understand optimism from a dispositional dimension, based on generalized expectations. Different theoretical and methodological assumptions of these theories are presented, as well as studies from both theories in different cultures and with other variables, such as self-esteem and personality.

Keywords: Optimism, Positive Psychology, personality, self-steem.
\end{abstract}

Del Optimismo Explicativo al Disposicional: la Perspectiva de la Psicología Positiva

\section{Resumen}

El optimismo desde la sabiduría popular, pasando por las discusiones filosóficas hasta los estudios empíricos en Psicología, está fuertemente asociado a una visión positiva de la vida, especialmente frente a las adversidades. El presente trabajo buscó presentar dos abordajes teóricos y de investigación empírica sobre el optimismo en el campo de la Psicología Positiva. La perspectiva de Seligman define el optimismo como aprendido, relacionado a los estilos explicativos, mientras que Scheier y Carver comprenden el optimismo a partir de una dimensión disposicional, basado en expectativas generalizadas. En el presente estudio se destacan las diferentes posiciones teóricas y metodológicas, los estudios realizados en diferentes culturas y con variables, como autoestima y personalidad.

Palabras clave: Optimismo explicativo, optimismo disposicional, Psicología Positiva.

\section{Introdução}

O presente trabalho visa apresentar um panorama sobre o estudo do otimismo no campo da Psicologa Positiva, a partir de duas abordagens teóricas e de investigação empírica. Estudos científicos demonstram que o otimismo está associado com a tomada de medidas proativas para proteger a saúde física e mental. Otimistas, quando confrontados com um desafio, tendem a agir com confiança e persistência, mesmo que o progresso seja difícil ou lento (Scheier, Carver, \& Bridges, 1994; Scheier \& Carver, 1985). Outros estudos apontam evidências de forte relação entre bem-estar e otimismo associado à alta autoestima e personalidade (Kam \& Meyer, 2012; Karademas, 2006).

A palavra otimismo vem do latim optimus que significa "o melhor". Para a sabedoria popular, é uma forma de ver as coisas pelo lado bom, de pensar positivo, de modo contrário ao pensamento negativo chamado pessimismo.

$\mathrm{Na}$ Filosofia, otimismo e pessimismo estão presentes nas obras de pensadores como Leibniz e Schopenhauer. Leibniz entendia o otimismo como a percepção segundo a qual a realidade é boa por sua própria natureza, consequentemente, o "bem" sempre vence o "mal". Considerado o principal adepto dessa tendência, Leibniz afirmava que "este Mundo é o melhor dos Mundos possíveis", ou seja, entre uma infinidade de mundos possíveis, há o melhor de todos, caso contrário Deus não o teria criado (Barbosa, 1997). O otimismo irracional do filósofo alemão recebeu forte crítica de Voltaire em seu livro "Cândido, ou o otimismo", no qual ironizou as ideias de Leibniz, chegando a rotulá-lo de ingênuo. 
Schopenhauer, por sua vez, não constituiu nenhuma escola filosófica do pessimismo, tão pouco teceu uma teoria específica sobre o tema, mas suas doutrinas foram rotuladas de pessimistas porque pensou a vontade como fonte de todo o sofrimento humano. Para o filósofo, embora a vontade fosse a raiz metafísica do mundo e da conduta humana, era algo sem nenhuma meta ou finalidade, um querer irracional e inconsciente que, inerente à existência humana, geraria dor e sofrimento. A felicidade seria apenas a interrupção temporária de um processo de infelicidade e somente a lembrança de um sofrimento passado criaria a ilusão do bem-estar presente (Ross-MacDonald,1984).

Já, na literatura, personagens clássicos como Cândido, ou o otimista (Voltaire, 1759) e Pollyanna (Porter, 1913) aparecem como representantes de um otimismo extremo. Cândido segue as ideias de seu mestre Dr. Pangloss que considerava que o mundo em que vivemos é o melhor dos mundos possíveis e, afirmava que todas as coisas são do jeito que são e não poderiam ser de outra maneira. Porém, ao término do romance, Cândido faz uma ressalva ao otimismo do Dr. Pangloss dizendo que, mesmo sendo as coisas como são, ainda assim, é necessário que haja um comprometimento do indivíduo com seus objetivos e sonhos. Pollyanna, por sua vez, por meio do jogo do contente, que havia aprendido com seu pai, não só procura ver o lado bom das coisas e acontecimentos, como incentiva a todos ao seu redor a fazer o mesmo. O otimismo expresso por esses personagens pode passar uma conotação de ingenuidade e de negação da realidade, mas, em última análise, traz em si a expectativa de eventos futuros positivos.

Tal conceito de otimismo emerge de pesquisas pioneiras como as de Carver e Scheier $(1982,1983)$ e Scheier e Carver (1985), nas últimas décadas do século XX, dentro do campo da Psicologia Positiva. Mesmo que muitos pesquisadores contemporâneos concordem com a concepção geral que o otimismo reflete uma expectativa de que coisas boas irão acontecer, enquanto que o pessimismo reflete uma expectativa de eventos ruins, não há consenso teórico e as discordâncias parecem estar na operacionalização dos construtos (Caver, Scheier, \& Segerstrom, 2010).

Snyder e Lopez (2002), em seu livro sobre Psicologia Positiva, destacam duas teorias que vem recebendo grande atenção com relação ao construto otimismo. São as teorias do Otimismo Aprendido de Martin Seligman e do Otimismo Disposicional de Michael Scheier e Charles Carver.

\section{Otimismo Aprendido ou Explicativo de Martin Seligman}

Para Seligman, ser otimista não se reduz a ter pensamentos positivos, mas ao modo como a pessoa pensa sobre as causas de eventos ruins (Seligman, 1998). A diferença entre otimistas e pessimistas reside na forma com que explicam a causa de eventos ruins ou bons que lhes acontecem no cotidiano, ou seja, como é seu "estilo explicativo" (explanatory style) (Peterson \& Steen, 2002).

A noção do estilo explicativo nasceu da ideia de desamparo aprendido. O modelo de desamparo aprendido, proposto primeiramente por Maier e Seligman (1976), demonstra que, após experienciar eventos aversivos incontroláveis, animais e pessoas desistem de evitar os estímulos ruins. Isso, possivelmente, porque aprenderam que não há contingência entre ações e resultados. Em estudo sobre o modelo do desamparo aprendido e depressão, Abramson, Seligman e Teasdale (1978) reformularam a ideia de desamparo associando esse construto ao modo como habitualmente as pessoas explicam eventos ruins, isto é, aos padrões de estilos explicativos.

Seligman (1998) descreve três dimensões dos estilos explicativos: Permanência, Difusão e Personalização. A Permanência se refere ao quanto os efeitos de determinado evento se prolongam no tempo, podendo ser estáveis ou temporários. A Difusão está relacionada à propagação dos efeitos do acontecimento para outras situações, podendo ser específica para determinada situação ou global, atingindo diferentes acontecimentos ou áreas da vida. Por fim, a personalização está associada ao quanto a causa do evento é atribuída a fatores externos ou internos.

Dentro dessas três dimensões, otimistas são pessoas que atribuem explicações permanentes, inespecíficas e internas para os eventos bons, e explicações temporárias, específicas e externas para eventos ruins. Enquanto que, pessimistas percebem os eventos bons como temporários, específicos e externos, não atribuindo ao seu esforço pessoal o acontecimento. Para os pessimistas, os eventos ruins são percebidos como permanentes, inespecíficos e internos.

Para Seligman et al., (1984) esse estilo explicativo de eventos possui raízes evolutivas nos componentes genético e ambiental. Do ponto de vista hereditário, estudos com gêmeos demonstram correlação mais elevada de otimismo aprendido em gêmeos monozigóticos (Schulman, Keith, \& Seligman, 1993). Porém, estudos com pais e filhos apontam para um forte vínculo entre ambiente e otimismo aprendido. Pais que ensinam as 
crianças a entenderem seus fracassos e os atribuírem a causas externas, temporárias e específicas, estão ensinando aos seus filhos a cultura adaptativa de encontrar desculpas e ter uma visão otimista dos acontecimentos (Snyder, Higgins, \& Stucky, 1983; 2005).

Para medir o estilo explicativo, em adultos, os pesquisadores desenvolveram o Attributional Style Questionnaire (ASQ) (Peterson et al., 1982). Esse questionário de autorrelato apresenta aos respondentes eventos hipotéticos que poderiam acontecer com eles e solicita que, para cada um dos eventos, o respondente indique qual é a melhor explicação para a causa deles nas dimensões dos estilos explicativos - interno-externa, estável/ transitória e global/específica. Para a análise final dos resultados, os escores dos eventos ruins e bons são combinados, embora tenham sido apontados separadamente no questionário.

Um segundo instrumento de medida foi desenvolvido por Peterson, Schulman, Castellon e Seligman (1992) o Content Analysis of Verbatim Explanations (CAVE). O CAVE permite acessar o estilo explicativo por meio da análise do conteúdo de textos ou discursos dos participantes. Os pesquisadores identificam, no material coletado, explicações para eventos ruins dadas pelos participantes e as apresentam para um grupo de juízes que as avaliam de acordo com as escalas do ASQ. A vantagem do CAVE é que elimina a problemática dos instrumentos de autorrelato e possibilita que se faça uma volta no tempo e se explore o otimismo/ pessimismo de figuras históricas por meio de suas falas, diários, cartas ou entrevistas.

Vários estudos vêm sendo realizados sobre otimismo aprendido na busca por um melhor entendimento do construto e seu impacto na vida das pessoas, por aprimoramento dos instrumentos de medida e construção de intervenções. Os resultados obtidos nos diferentes estudos demonstram, por exemplo, consistência entre otimismo e melhor desempenho no trabalho, nos estudos, nos relacionamentos e no enfrentamento de situações adversas. Assim como apontam alta correlação entre pessimismo e doenças físicas e mentais (Abramson et al., 2000; Meyer, 1988; Seligman, 1998).

Seligman (1998), em seu livro intitulado Learned optimism, apresenta um estudo realizado com a técnica CAVE. Foram coletados os comentários dos jogadores de duas equipes da National League (USA) nas entrevistas dadas ao jornal Sporting News e nas seções de esportes de jornais locais durante os seis meses da temporada de 1985. Foram analisadas 15.000 páginas.
Como resultados, observou-se que os jogadores do Mets transmitiam um estilo explicativo otimista em suas falas, enquanto os St. Louis Cardinals apresentavam um estilo pessimista. Esses modelos explicativos foram usados para predizer o desempenho na temporada seguinte. Os comentários otimistas dos Mets sugeriam êxito ao time, enquanto que os comentários pessimistas do outro time sugeriam fracasso, e foi exatamente o que aconteceu na temporada de 1986. Como os pesquisadores estavam ainda céticos em relação aos resultados, replicaram o estudo no ano de 1986 para predizer o desempenho em 1987 e confirmaram os resultados.

Peterson, Seligman e Vaillant (1988) realizaram um estudo com 99 estudantes do sexo masculino das classes de 1942-1944, que participavam de um estudo longitudinal sobre desenvolvimento em adultos da Universidade de Harvard. Por meio da técnica CAVE, foram analisadas as respostas dadas pelos participantes em um questionário semiestruturado sobre experiências difíceis na guerra. Os resultados demonstraram que homens que explicavam eventos ruins como estáveis, globais e internos, aos 25 anos de idade, eram menos saudáveis três décadas depois que homens que atribuíam tais eventos a causas instáveis, inespecíficas e externas. Os pesquisadores concluíram que pessoas que, no início da vida adulta, habitualmente, atribuem aos eventos negativos causas estáveis, globais e internas apresentam risco de saúde na meia idade.

Hirsch, Wolford, LaLonde, Brunk e Morris (2009) realizaram um estudo com o objetivo de examinar o efeito moderador do estilo explicativo sobre a relação entre experiências negativas de vida e ideação suicida em uma amostra de 138 estudantes de uma universidade localizada em área rural nos Estados Unidos. Como resultados, os pesquisadores encontraram que o estilo explicativo otimista minimiza a influência dos eventos de vida negativos e traumáticos que potencializam a incidência de pensamentos de suicídio, para além dos efeitos de desesperança e depressão. Assim, o estilo explicativo pessimista foi associado com pensamentos suicídas, enquanto que o estilo explicativo otimista foi associado à ausência ou baixos níveis de ideação suicida.

\section{Otimismo Disposicional de Scheier e Carver}

Para Scheier e Carver (1985; Scheier et al., 1994) as definições de otimismo e pessimismo repousam sobre as expectativas que as pessoas possuem sobre eventos futuros. Assim, otimistas são pessoas que esperam que coisas boas aconteçam, enquato pessimistas são pessoas que esperam que coisas ruins aconteçam. 
Essas expectativas generalizadas de resultados podem envolver percepções em relação a ser capaz de avançar em direção a objetivos desejáveis ou se afastar dos não desejáveis (Carver \& Scheier, 1998).

Tal concepção está ancorada no expectancy-value model of motivation, para qual o comportamento reflete a busca por objetivos que são ações ou estados desejados e, acredita-se possíveis de serem alcançados (Carver \& Scheier, 1998; Carver et al., 2010). Existem dois elementos importantes nessa equação: as expectativas positivas e o senso de confiança. Para que a pessoa empenhe esforços no sentido de alcançar um objetivo, é preciso que tenha expectativas generalizadas positivas com relação ao evento futuro. Bem como um forte senso de confiança no desfecho positivo. Se faltar convicção, não haverá ação ou os esforços serão interrompidos. Apenas quando a confiança é suficientemente alta, a pessoa age e continua empenhando esforços na direção do objetivo (Scheier \& Carver, 1993).

Dentro do modelo expectancy-value, o elemento que define o otimismo disposicional são as expectativas. As expectativas existem em muitos níveis e variam ao longo do ciclo vital, podendo ter uma natureza específica ou mais geral (Carver \& Scheier, 2003). Por exemplo, um adolescente pode ter a expectativa de aprender a dirigir seu carro para ir visitar seus amigos, expectativa de passar no vestibular para a faculdade desejada e a expectativa de se sentir feliz em sua vida adulta.

As expectativas são permeadas pelo senso de convicção ou incerteza com relação aos eventos futuros. Assim, otimistas, quando confrontados com um desafio, tendem a agir com confiança e persistência, mesmo que o progresso seja difícil ou lento. Já pessimistas tendem a ser indecisos e hesitantes. $\mathrm{E}$ as diferenças entre otimismo e pessimismo são ampliadas quando surgem obstáculos e sinistros. Otimistas presumirão que as adversidades poderão ser superadas com facilidade ou com o empenho de um esforço adicional, porém, talvez não tenham claro quando é necessário desistir (Janoff-Bulman \& Brickman, 1982). Pessimistas, em geral, tendem a antecipar dificuldades e a não acreditar na possibilidade de superação delas (Scheier, Carver, \& Bridges, 2001).

Para medir o otimismo, Scheier e Carver (1985), primeiramente, propuseram o Life Orientation Test (LOT), o qual incluia expectativas positivas e negativas. O LOT foi assumido como uma medida unidimensional, na qual otimismo e pessimismo representam polos opostos de um continuum. Diversas pesquisas que foram realizadas posteriormente apontaram para resultados controversos com relação à unidimensionalidade do construto. Também indicaram coincidências com Neuroticismo, Ansiedade Traço, Domínio de Si Próprio e Autoestima (Chang, D’Zurilla, \& Maydeu-Olivares, 1994; Dember \& Brooks, 1989; Smith, Pope, Rhodewalt, \& Poulton, 1989). Devido às referidas críticas Scheier, Carver e Bridges (1994) propuseram a revisão do instrumento e, ao retirarem itens que não focavam explicitamente em expectativas com relação ao futuro, criaram o Revised Life Orientation Test (LOT-R), eliminando as possíveis coincidências com outros construtos.

Porém, a controvérsia com relação à dimensionalidade do teste continua. No estudo de revisão do LOT, os pesquisadores realizaram dois tipos de análises fatoriais (Varimax e Oblimin) e chegaram à mesma solução de um fator. Realizaram, também, análise fatorial confirmatória e as diferenças encontradas entre a solução de um fator e dois não foram significativas. Portanto, os autores assumiram o teste como unidimensional. Estudos posteriores corroboram a ideia de um único fator (Bandeira, Bekou, Lott, Teixeira, \& Rocha, 2002; Chang \& McBride-Chang, 1996; Lai, Cheung, Lee, \& Yu, 1998), porém, outros estudos apontam que o LOT-R seria bidimensional, sendo otimismo e pessimismo fatores independentes (Gaspar, Ribeiro, Matos, Leal, \& Ferreira, 2009; Hummer, Dember, Melton, Howe, \& Schefft, 1992; Reilley, Geers, Lindsay, Deronde, \& Dember, 2005)

No Brasil, foi realizado estudo de adaptação e validação do teste LOT-R (Bastianello, Pacico, \& Hutz, 2014) a partir do original americano. Os pesquisadores encontraram uma única dimensão e correlações positivas entre otimismo e autoestima. Anteriormente, uma adaptação e validação desse instrumento a partir de uma versão francesa já haviam sido feita (Bandeira et al., 2002).

Essas diferenças dimensionais revelam um ponto sensível da teoria do Otimismo Disposicional. Seriam otimismo e pessimismo polos opostos de um continuum ou construtos distintos que coexistem em diferentes níveis e intensidade? Certamente mais estudos sobre as evidências de validade do teste, com diferentes amostras e em outras culturas ampliarão as discussões e apontarão para a melhor definição.

Considerando-se as duas teorias sobre otimismo apresentadas, evidenciam-se as diferenças no conceito e, por consequência, na abordagem do referido construto. Entender o otimismo como disposicional ou como um modo explicativo sobre os eventos futuros 
implica na construção de diferentes instrumentos de avaliação e em intervenções distintas.

Os estudos do otimismo no campo da Psicologia Positiva começaram há, aproximadamente, duas décadas atrás, o que em ciência é um tempo relativamente pequeno para a solidificação de uma teoria por meio de evidências científicas. O panorama que existe atualmente na literatura aponta para a necessidade do desenvolvimento de mais pesquisas sobre otimismo, seja na busca por um melhor entendimento sobre o seu conceito, seja no estudo das suas correlações com indicadores positivos do desenvolvimento.

Alguns estudos já demonstram que o otimismo não deve ser entendido como uma variável isolada, mas correlacionado, especialmente, com fatores de personalidade e indicadores positivos do desenvolvimento, como autoestima. Com o objetivo de tornar mais clara a correlação do otimismo com outras variáveis, serão apresentados, a seguir, alguns estudos que vêm sendo desenvolvidos na área.

\section{Otimismo e Autoestima}

A autoestima é o senso de valor próprio que o indivíduo carrega, tendo a convicção que será particularmente mais aceito que rejeitado, e que não é um fracasso em sua vida (Coopersmith, 1967; Crocker \& Major, 1989; Hutz \& Zanon, 2011; Rosenberg, 1965,). Estudos sugerem que a autoestima aumenta a motivação (Aspinwall \& Taylor, 1992) e está relacionada ao maior nível global de persistência em objetivos direcionados (Di Paula \& Campbell, 2002). Além de aumentar a probabilidade de sucesso, a autoestima elevada, também, pode diminuir o efeito do fracasso ocasional e, assim, diminuir o risco do desenvolvimento de níveis elevados de pessimismo (Campbell, Chew, \& Scratchley, 1991).

Scheier e Carver (1993) sugerem que o otimismo é parcialmente aprendido por meio de experiências anteriores de sucesso e fracasso. Tais experiências estão entre os principais preditores de diferenças individuais em autoestima (Harter, 1993). Estudos empíricos, também, demonstram alta correlação entre autoestima e otimismo, variando de 0,67 em adolescentes (Carvajal, Clair, Nash, \& Evans, 1998), 0,62 entre homens e mulheres HIV positivo (Andersson, 1999) e 0,80 em mulheres grávidas, entre 20 e 43 anos (Fontaine \& Jones, 1997). Desse ponto de vista, a explicação para a associação entre a autoestima e otimismo pode ser o papel potencial da autoestima em aumentar a possibilidade de experiências de sucesso.
Heinonen, Raikkonen e Keltikangas-Jarvinen (2005) realizaram um estudo longitudinal de 21 anos com adolescentes participantes de um programa para jovens finlandeses com riscos cardiovasculares. Aos 12 (T1) e 18 anos (T2) os participantes responderam o inventário de Autoestima de Coopersmith e, aos 33 anos, o teste LOT-R. Os resultados demonstraram que os adolescentes cuja autoestima manteve-se baixa ou alterou-se (escores mais altos ou mais baixos) do T1 para o T2 apresentaram altos escores de pessimismo aos 33 anos de idade. Não foram encontradas diferenças entre sexo. Como conclusão, os pesquisadores observaram que existe correlação positiva entre altos níveis de autoestima e uma orientação otimista para vida.

Não há ainda uma vasta literatura sobre a relação entre otimismo e autoestima, mas os estudos já apontam para a forte vinculação dessas variáveis. Tal constatação fornece subsídios, para pesquisadores e profissionais, na busca por evidências e intervenções nos aspectos positivos dos seres humanos, para o entendimento da importância da influência da correlação do otimismo e da autoestima na saúde física e mental dos indivíduos.

\section{Otimismo e Personalidade no Modelo dos Cinco Grandes Fatores}

Pode-se dizer que personalidade é uma organização dinâmica de sistemas psicológicos internos que determinam disposições e traços característicos de comportamento, pensamento e sentimento. É um conjunto de características que constituem a identidade de uma pessoa e a torna única devido a sua continuidade, estabilidade e consistência ao longo do tempo (Eysenck, 1947). Embora exista uma infinidade de traços, há uma longa tradição de estudos de personalidade que demonstram que a estrutura básica da personalidade consiste em cinco fatores designados os Cinco Grandes Fatores (CGF) ou big five (Nunes, Hutz, \& Nunes, 2010).

O modelo CGF desenvolveu-se a partir de pesquisas realizadas na área das teorias fatoriais e de traços de personalidade (Cattell, 1947; Eysenck, 1947; McCrae \& Costa, 1997; Nunes et al., 2010; Tupes \& Christal, 1961/1992;). Não há uma explicação a priori para e eleição dos cinco fatores, uma vez que a descoberta deles se constitui em uma generalização empírica, que foi replicada, independentemente, inúmeras vezes. O modelo CGF tem suas origens na análise da linguagem utilizada para descrever pessoas. $\mathrm{O}$ uso de adjetivos da linguagem natural como descritores de traços tem sido defendido como a melhor estratégia para identificar 
fatores que permitam entender melhor as características de personalidade (Briggs, 1992). Os CGF incluem as dimensões Neuroticismo, Extroversão, Socialização, Realização e Abertura (Nunes et al., 2010).

Pesquisas correlacionando os fatores de personalidades e o otimismo apontam que o pessimismo está associado com Neuroticismo e afetos negativos, enquanto que o otimismo relaciona-se com Extroversão e afetos positivos, tendo otimismo e pessimismo como dois construtos distintos (Marshall, Wortman, Kusulas, Hervig, \& Vickers, 1992). Estudos dessa natureza inserem-se na discussão sobre a dimensionalidade do construto otimismo e evidenciam as implicações teóricas e práticas que, ainda, os pesquisadores precisam responder para se avançar no entendimento das relações entre personalidade e otimismo.

Sharpe, Martin e Roth (2011), ao conduzirem uma pesquisa utilizando três tipos de instrumentos de medida de otimismo e cinco para medir personalidade no modelo dos CGF, encontraram resultados que demonstram que o otimismo disposicional está fortemente relacionado com quatro fatores de personalidade (Neuroticismo, Extroversão, Socialização e Realização). Embora Neuroticismo e Extroversão tenham apresentado relações mais fortes com otimismo, os fatores Socialização e Realização representaram variância adicional significativa na predição do otimismo. Os pesquisadores concluíram que, aparentemente, o otimismo é potencialmente mais que apenas baixo. Neuroticismo, ou uma faceta da Extroversão é uma variável complexa que faz parte da personalidade e que necessita mais investigações.

Internacionalmente, há um maior volume de pesquisas relacionando personalidade no modelo dos CGF e otimismo. No Brasil, os estudos ainda são poucos, mas os resultados encontrados já se somam aos achados da literatura estrangeira. Em recente pesquisa com adolescentes e adultos brasileiros (Bastianello, 2014) encontrou-se, como esperado, correlação negativa entre Neuroticismo e otimismo, e correlação positiva com os demais fatores de personalidade (Extroversão, Socialização, Realização e Abertura) tanto em adolescentes quanto em adultos. Com relação à influência da personalidade sobre o otimismo, observou-se que, em adolescentes, a preponderância da personalidade sobre uma visão positiva do futuro é maior que em adultos. Tal achado, possivelmente, deve-se ao fato dos adolescentes possuírem menos experiências de vida que um adulto, sendo assim, seu senso de confiança no futuro é mais elevado. Para adultos, mais que a personalidade, provavelmente, fatores situacionais, como estilo de vida, estado civil, ambiente de trabalho e experiências já vividas tenham maior impacto sobre o otimismo.

Em síntese, com o acúmulo de evidências, fica claro que otimismo e personalidade possuem forte correlação, mas ainda são necessários estudos que clarifiquem a utilidade preditiva diferencial de otimismo e pessimismo com relação à Neuroticismo e à Extroversão, bem como a relação com os demais fatores de personalidade.

\section{Beneficios do Otimismo}

Seres humanos, ao serem confrontados com situações difíceis, adversas ou traumáticas experimentam uma gama de sentimentos desde ansiedade, raiva, depressão a entusiasmo, força e superação. $\mathrm{O}$ balanço entre sentimentos positivos e negativos está fortemente relacionado às diferenças entre as orientações otimista e pessimista na vida (Carver et al., 2010).

Diversos estudos demonstram que ser otimista quando os tempos são difíceis possibilita que a pessoa vivencie menor ansiedade, mantenha-se empreendendo esforços contínuos e crie estratégias em direção a melhores resultados (Brissette, Scheier, \& Carver, 2002; Segerstrom, 2007; Shnek, Irvine, Stewart, \& Abbey, 2001; Solberg Nes, Evans, \& Segerstrom, 2009). Dado o acúmulo de evidências, parece claro que o otimismo é uma variável de diferença individual, que desempenha um papel central nas experiências humanas.

O otimismo tem sido associado a melhores resultados em recuperação em diversas áreas da saúde física. Zenger, Brix, Borowski, Stolzenburg e Hinz (2010) realizaram um estudo na Alemanha com pacientes com câncer urogenital durante a estadia no hospital (T1), duas semanas mais tarde (T2) e três meses depois (T3). Após avaliarem os níveis de ansiedade, depressão e qualidade de vida, os pesquisadores encontraram que o otimismo em T1 estava significativamente associado a essas variáveis e era preditor delas nos tempos seguintes (T2 e T3). Concluíram que os doentes com um baixo nível de otimismo e um alto nível de pessimismo apresentavam maior risco para elevados graus de ansiedade e depressão, além de apresentarem baixa qualidade de vida.

As vantagens de uma orientação otimista para a vida também parecem estar presentes no domínio das relações interpessoais, uma vez que pessoas otimistas, com sua tendência a perceber os eventos de vida da melhor forma possível, acabam envolvendo mais esforços para a solução de problemas que mantêm os 
relacionamentos vivos. Türküm (2005), ao investigar otimismo, comportamento submisso, riqueza da rede social e variáveis demográficas como preditoras de bem-estar psicológico, em uma amostra da população turca, encontrou forte associação entre as variáveis. Os resultados do estudo apontam que ser casado, ter uma profissão e ser otimista possui impacto positivo na percepção do bem-estar subjetivo.

Outro aspecto que vem sendo bastante associado às diferenças individuais entre otimistas e pessimistas são as estratégias de enfrentamento utilizadas por ambos. Otimistas são confiantes com relação ao sucesso na realização de suas metas, portanto continuam empreendendo esforços mesmo diante de adversidades. Já pessimistas não possuem confiança suficiente no sucesso e tendem a escapar das dificuldades por meio de distrações, tendendo a interromper seus esforços precocemente. Em um estudo realizado no Reino Unido com atletas, observou-se a relevância dos níveis de otimismo no emprego de melhores estratégias de enfrentamento em resistência mental. Os resultados demonstram que os níveis mais elevados de resistência mental são apresentados por atletas otimistas e estão associados com maior utilização de estratégias de enfrentamento do tipo imagens mentais positivas, empreendimento de mais esforços, controle do pensamento e análise lógica das situações (Nicholls, Polman, Levy, \& Backhouse, 2008).

Em suma, já existem diversas evidências empíricas demonstrando que uma orientação otimista para vida está fortemente associada a maior bem-estar subjetivo, estratégias de enfrentamentos eficazes e maiores cuidados com a saúde, trabalho e relacionamentos. Ser otimista é preditor de melhor saúde física e mental, e de recursos mais adaptativos diante das adversidades nos diferentes âmbitos da vida (Scheier \& Carver, 1992).

\section{Consideração Final}

O otimismo do ponto de vista da sabedoria popular, passando pelas discussões filosóficas até os estudos científicos em Psicologia, está fortemente associado a uma visão positiva da vida, especialmente diante dos eventos difíceis e disruptivos. Pessoas com uma disposição otimista sustentam expectativas generalizadas positivas de êxito e realização no futuro, mesmo que enfrentem grandes dificuldades ou fracassos. Um crescente número de estudos sustenta a ideia que o otimismo confere mais benefícios nos âmbitos intra e interpessoais que o pessimismo.
Mesmo que, em algumas situações, o otimismo possa levar a pessoa a ter uma visão distorcida de suas capacidades e possibilidades em atingir a meta desejada, assim como certo grau de pessimismo defensivo sinaliza comportamentos mais parcimoniosos, uma orientação otimista para a vida parece conferir benefícios superiores.

Muitos estudos já foram produzidos internacionalmente nas últimas duas décadas sobre o otimismo, contudo os pesquisadores apontam que mais pesquisas são necessárias para ajudar a responder questões como a estrutura dimensional do otimismo: seria o otimismo e pessimismo polos opostos de um mesmo contínuo ou construtos separados que constituem uma estrutura bipolar? Pesquisas, também, são necessárias sobre os antecedentes desenvolvimentais do otimismo, especialmente sobre as influências ambientais.

Estudos já apontam a influência do otimismo na constituição da personalidade e de indicadores positivos, como autoestima. Uma vez que essa relação já foi provada em diversos estudos, futuras investigações no sentido de se identificar os mecanismos neurofisiológicos envolvidos que contribuem para diferenças no bem-estar físico e mental têm sido recomendadas por pesquisadores da área.

Por fim, alguns estudos sobre otimismo já apontam para progressos na área de intervenções clínicas, educacionais e organizacionais. No entanto, muito há ainda para ser pesquisado no sentido da construção de intervenções eficientes.

Considerando-se o panorama das pesquisas sobre otimismo no Brasil, encontramos poucos estudos na área sendo um campo propício para os pesquisadores. Já conta-se com instrumentos para medir otimismo adaptados e validados para a população brasileira e pesquisas sendo desenvolvidas no campo da saúde, com pacientes clínicos, na área organizacional, sobre engajamento, liderança autêntica e escolha profissional, dentre outros.

Com o acúmulo de evidências, fica claro que o otimismo é uma variável de diferença individual que desempenha um papel central nas experiências humanas. Do ponto de vista da personalidade, o traço otimista-pessimista compõe importantes fatores, como Neuroticismo e Extroversão. De um ponto de vista clínico, contudo, o desafio está em se conhecer mais profundamente os mecanismos e processos do otimismo, os quais caracterizam a forma com que a pessoa otimista compreende os eventos futuros. E a partir daí, investigar se há possibilidade de se ensinar essa forma de abordagem do mundo para os pessimistas. 


\section{Referências}

Abramson, L. Y., Alloy, L., Hankin, B, Clements, C., Zhu, L., \& Hogan, M., (2000). Optimistic cognitive style and invulnerability to depression. In J. Gillham (Ed.), The science of optimism and hope (pp. 75-98). Philadelphia, PA: Templeton Foundation Press.

Abramson, L. Y., Seligman, M. E. P., \& Teasdale, J. D. (1978). Learned helplessness in humans: Critique and reformulation. Journal of Abnormal Psychology, 87, 49-74.

Andersson, G. (1999). Anxiety, optimism and symptom reporting following surgery for acoustic neuroma. Journal of Psychosomatic Research, 46, 257-260.

Aspinwall, L. G., \& Taylor, S. E. (1992). Modeling cognitive adaptation: A longitudinal investigation of the impact of individual differences and coping on college adjustament and performance. Journal of Personality and Social Psychology, 61, 755-765.

Bandeira, M., Bekou, V., Lott, K., Teixeira, M., \& Rocha, S. (2002). Validação transcultural do teste de orientação da vida (TOV-R). Estudos de Psicologia, 7(2), 251-258.

Barbosa, J. (1997). Schopenhauer: A decifração do enigma do mundo. São Paulo, Brasil: Moderna.

Bastianello, M. R. (2014). Otimismo e personalidade: o contexto brasileiro. Mesa redonda no I Congresso Brasileiro de Psicologia Positiva, Porto Alergre, RS.

Bastianello, M. R., Pacico, J. C., \& Hutz, C. S. (2014). Optimism, self-esteem and personality: adaptation and validation of the Brazilian version of the Revised Life Orientation Test (LOT-R). PsicoUSF, 19(3), 523-531.

Briggs, S. R. (1992). Assessing the five-factor model of personality description. Journal of Personality, 60, 253-293.

Brissette, I., Scheier, M. F., \& Carver, C. S. (2002). The role of optimism in social network development, coping, and psychological adjustment during a life transition. Journal of Personality and Social Psychology, 82, 102-111.

Campbell, J. D., Chew, B., \& Scratchley, L. S. (1991). Cognitive and emotional reactions to daily events: The effects of self-esteem and self complexity. Journal of Personality, 59, 473-505.
Carvajal, S. C., Clair, S. D., Nash, S. G., \& Evans, R. I. (1998). Relating optimism, hope, and self-esteem to social influences in deterring substance use in adolescents. Journal of Social and Clinical Psychology, 17, 443-465.

Carver, C. S., \& Scheier, M. F. (1982). Outcome expectancy, locus of attribution for expectancy, and self-directed attention as determinants of evaluations and performance. Journal of Experimental Social Psychology, 18, 184-200.

Carver, C. S., \& Scheier, M. F. (1983). A control-theory approach to human behavior and implications for self-management. In P. C. Kendall (Ed.), Advances in cognitive-behavioral research and therapy, (pp. 127194). New York: Academic.

Carver, C. S., \& Scheier, M. F. (1998). On the self-regulation of behavior. New York: Cambridge University Press.

Carver, C. S., \& Scheier, M. F. (2003). Optimism. In J. L. Shane \& C. R. Snyder, Positive psychology assessment: $A$ handbook of models and measures (pp 75-89). Washington, DC: American Psychological Association.

Carver, S. C., Scheier, M. F., \& Segerstrom, S.C. (2010). Optimism. Clinical Psychology Review, 30, 879-889.

Cattell, R. B. (1947). Confirmation and clarification of primary personality factors. Psychometrika, 12, 197-220.

Chang, E. C., D’Zurilla, T. J., \& Maydeu-Olivares, A. (1994). Assessing the dimensionality of optimism and pessimism using a multimeasure approach. Cognitive Therapy and Research, 18, 143-160.

Chang, L., \& Mcbride-Chang, C. (1996). The factor structure of the life orientation test. Educational and Psychological Measurement, 56(2), 325-329.

Coopersmith, S. (1967). The Antecedents of Self-Esteem. San Francisco: W.H. Freeman.

Crocker, J., \& Major, B. (1989). Social stigma and selfesteem: The self-protective properties of stigma. Psychological Review, 96, 608-630.

Dember, W. N., \& Brooks, J. (1989). A new instrument for measuring optimism and pessimism: Testretest reliability and relations with happiness and religious commitment. Bulletin of Psychometric Society, 27, 365-366. 
Di Paula, A., \& Campbell, J. D. (2002). Self-esteem and persistence in the face of failure. Journal of Personality and Social Psychology, 83, 711-724.

Eysenck, H. J. (1947). Dimensions of personality. New York, NY: Praeger.

Fontaine, K. R., \& Jones, L. C. (1997). Self-esteem and optimism, and postpartum depression. Journal of Clinical Psychology, 53, 59-63.

Gaspar, T., Ribeiro, J. L. P., Matos, M. G., Leal, I., \& Ferreira, A. (2009). Optimismo em crianças e adolescentes: Adaptação e validação do LOT-R. Psicologia: Reflexão e Crítica, 22(3), 439-446.

Harter, S. (1993). Causes and consequences of low self-esteem in children and adolescents. In R. F. Baumeister (Ed.), The puzile of low self-regard (pp. 87-116). New York: Plenum.

Heinonen, K., Raikkonen, K., \& Keltikangas-Jarvinen, L. (2005). Self-esteem in early and late adolescence predicts dispositional optimism-pessimism in adulthood: A 21-year longitudinal study. Personality and Individual Differences, 39, 511-521

Hirsch, J. K., Wolford, K., LaLonde, S. M., Brunk, L., \& Morris, A. P. (2009). Optimistic explanatory style as a moderator of the association between negative life events and suicide ideation. Crisis - The Journal of Crisis Intervention and Suicide Prevention, 30(1), 48-53

Hummer, M., Dember, W. N., Melton, R. S., Howe, S. R., $\&$ Schefft, B. (1992). On the partial independence of optimism and pessimism. Current Psychology: Research and Reviews, 11, 37-50.

Hutz, C. S., \& Zanon, C.(2011). Revision of the adaptation, validation, and normatization of the Roserberg self-esteem scale. Avaliação Psicológica, 10(1), 41-49.

Janoff-Bulman, R., \& Brickman, P. (1982). Expectations and what people learn from failure. In N. T. Feather (Ed.), Expectations and actions: Expectancyvalue models in psychology (pp.207-237). Hillsdale, NJ: Lawrence Erlbaum Associates Inc.

Kam, C., \& Meyer, J. P. (2012). Do optimism and pessimism have different relationships with personality dimensions? A re-examination. Personality and Individual Differences, 52(2), 123-127.

Karademas, E. C. (2006). Self-efficacy, social support and well-being: The mediating role of optimism. Personality and individual differences, 40(6), 1281-1290.

Lai, J., Cheung, H., Lee, W., \& Yu, H. (1998). The utility of the revised life orientation test to measure optimism among Hong Kong Chinese. International Journal of Psychology, 33(1), 45-56

Maier, S. F., \& Seligman, M. E. P. (1976). Learned helplessness: Theory and evidence. Journal of Experimental Psychology: General, 105, 3-46.

Marshall, G. N., Wortman, C. B., Kusulas, J. W., Hervig, L. K., \& Vickers Jr., R. R. (1992). Distinguishing optimism from pessimism: Relations to fundamental dimensions of mood and personality. Journal of Personality and Social Psychology, 62, 1067-1074.

Meyer, D. (1988). The positive thinkers: Popular religious psychology from Mary Baker Eddy to Nornlan Vincent Peale and Ronald Reagan (rev.). Middletown, CT: Wesleyan University Press.

McCrae, R. R., \& Costa, P. T. (1997). Personality Trait Sructure as a Human Universal. American Psychologist, 52, 509-516.

Nicholls, A. R., Polman, R. C. J., Levy, A. R., \& Backhouse, S. H. (2008). Mental toughness, optimism, pessimism, and coping among athletes. Personality and Individual Differences, 44, 1182-1192.

Nunes, C. H. S. S., Hutz, C. S., \& Nunes, M. F. O (2010). Bateria fatorial de personalidade (BFP). São Paulo: Casa do Psicólogo.

Peterson, C., Semmel, A., yon Baeyer, C., Abramson, L. Y., Metalsky, G. I., \& Seligman, M. E. P. (1982). The attributional style questionnaire. Cognitive Therapy and Research, 6, 287-299.

Peterson, C., Seligman, M. E. P., \& Vaillant, G. E. (1988). Pessimistic explanatory style is a risk factor for physical illness: A thirty-five year longitudinal study. Journal of Personality and Social Psychology, 55, 23-27.

Peterson, C., Schulman, P., Castellon, C., \& Seligman, M. E. P. (1992). CAVE: Content analysis of verbatim explanations. In C. P. Smith (Ed.), Motivation and personality: Handbook of thematic content analysis (pp. 383-392). New York: Cambridge University Press.

Peterson, C., \& Steen, T.A. (2002). Optimisc explanatory style. In C. R. Snyder \& S. J. Lopez (Eds.), The handbook of positive psychology (pp.231-243). New York: Oxford University Press. 
Porter, E. H. (1913). Pollyanna. Retirado de www.literaturepage.com/read/pollyanna.html

Reilley, S., Geers, A., Lindsay, D., Deronde, L., \& Dember, W. (2005). Convergence and predictive validity in measures of optimism and pessimism: Sequencial studies. Current Psychology, 24(1), 43-59.

Rosenberg, M. (1965). Society and the adolescent self-image Princeton, NJ: Princeton University Press.

Ross-MacDonald, G. (1984). Leibnir: New York: Oxford University Press.

Scheier, M. F., \& Carver, C. S. (1985). Optimism, coping, and health: Assessment and implication of generalized outcome expectancies. Health Psychology, 4, 219-247.

Scheier, M. F., \& Carver, C. S. (1992). Effects of optimism on psychological and physical well-being: Theoretical overview and empirical update. Cognitive Therapy and Research, 16, 201-228.

Scheier, M. F., \& Carver, C. S. (1993). On the power of positive thinking: The benefits of being optimistic. Current Directions in Psychological Science, 2, 26-30.

Scheier, M. F., Carver, C. S., \& Bridges, M. W. (1994). Distinguishing optimism from neuroticism (and trait anxiety, self-mastery, and self-esteem): A reevaluation of the life orientation test. Journal of Personality and Social Psychology, 67, 1063-1078.

Scheier, M. F., Carver, C. S., \& Bridges, M. W. (2001). Optimism, pessimism, and psychological well-being. In E. C. Chang (Ed.), Optimism and pessimism: Implications for theory, research, and practice (pp. 189216). Washington, DC: American Psychological Association.

Scheier, M.F., Carver, C.S., \& Segerstrom, S.C. (2010). Optimism. Clinical Psychology Review, 30, 879-889.

Schulman, P., Keith, D., \& Seligman, M. E. (1993). Is optimism heritable? A study of twins. Behaviour research and therapy, 31(6), 569-574.

Segerstrom, S. C. (2007). Optimism and resources: Effects on each other and on health over 10 years. Journal of Research in Personality, 41, 772-786.

Seligman, M. E. P., Peterson, C., Kaslow, N. J., Tanenbaum, R. J., Alloy, L. B., \& Abramson, L. Y. (1984). Attributional style and depressive symptoms among children. Journal of Abnormal Psychology, 83, 235-238.
Seligman, M. E. P. (1998). Learned optimism: How to change your mind and your life. New York: Free Press.

Sharpe, J. P., Martin, N. R., \& Roth, K. A. (2011). Optimism and the big five factors of personality: Beyond neuroticism and extraversion. Personality and Individual Differences, 51, 946-951.

Shnek, Z. M., Irvine, J., Stewart, D., \& Abbey, S. (2001). Psychological factors and depressive symptoms in ischemic heart disease. Health Psychology, 20, 141-145.

Smith, T. W., Pope, M. K., Rhodewalt, F., \& Poulton, J. L. (1989). Optimism, neuroticism, coping and symptom reports: An alternative interpretation of the life orientation test. Journal of Social and Clinical Psychology, 56, 640-648.

Snyder, C. R., \& Lopez, S. J. (2002). Psicologia positiva: Uma abordagem cientifica e prática das qualidades humanas. Porto Alegre: Artmed.

Snyder, C. R., Higgins, R. L., \& Stucky, R. J. (1983/2005). Excuses: Masquer ades in search of grace. New York: Wiley. Republished 2005 by perchevon Press, Clinton Corners, NY.

Solberg Nes, L., Evans, D. R., \& Segerstrom, S. C. (2009). Optimism and college retention: Mediation by motivation, performance, and adjustment. Journal of Applied Social Psychology, 39, 1887-1912.

Tupes, E. C., \& Christal, R. E. (1992). Recurrent personality factors based on trait ratings. Journal of Personality, 60, 225-252.

Türküm, A. S. (2005). Do optimism, social network richness, and submissive behaviors predict wellbeing? Study with a Turkish sample. Social Behavior and Personality, 33(6), 619-628.

Voltaire, C. (1759). Cândido. Retirado de http://www. ebooksbrasil.org/eLibris/candido.html

Zenger, M., Brix, C., Borowski, J., Stolzenburg, J., \& Hinz, A. (2010). The impact of optimism on anxiety, depression and quality of life in urogenital cancer patients. Psycho-Oncology, 19, 879-886.

Recebido: $12 / 08 / 2014$ $1^{a}$ reformulação: 30/10/2014 $2^{a}$ reformulação: 13/12/2014

Aprovado: 15/01/2015 
Sobre os autores:

Micheline Roat Bastianello é psicóloga pela Universidade Federal de Santa Maria (UFSM), mestre e doutora em Psicologia pela Universidade Federal do Rio Grande do Sul (UFRGS).

Claudio Simon Hutz é professor titular na Universidade Federal do Rio Grande do Sul (UFRGS). Suas linhas de pesquisa estão focadas em Psicologia Positiva, Avaliação Psicológica e Psicometria e Desenvolvimento Social e da Personalidade.

Contato com os autores:

Rua Ramiro Barcelos, 2600 - sala 101

CEP: 90035003

E-mail: mbastianello@hotmail.com 